\title{
Numerical shape optimization as an approach to reduce material waste in injection molding
}

\author{
Mario Studer • Frank Ehrig
}

Received: 28 July 2014 / Accepted: 22 December 2014 / Published online: 11 January 2015

(C) Springer-Verlag London 2015

\begin{abstract}
Reducing material waste is a major issue during new product development, especially for injection-molded parts, where the wall thickness has a strong influence on molding times and therefore on the manufacturing cost. Finding the minimum material amount for producing highquality products is a difficult and time-consuming task using state-of-the-art computer-aided engineering CAE tools. In this study, a numerical procedure is presented to reduce the material amount required for injection-molded parts by optimizing their wall thickness distributions with respect to part quality and identifying an upper limit for the injection pressure. The closed-loop procedure consists of three main parts: (1) a mesh parameterization tool for manipulating the thickness distribution of the part, (2) a mold flow analysis for evaluating the producibility and part quality, and (3) a genetic algorithm for adjusting the design variables toward minimum material waste. The effectiveness of the procedure is demonstrated into two different industrial parts. Using the part volume as the main objective, reductions in material waste of approximately 25 to $30 \%$ can be achieved via a slight improvement in part warpage compared to the initial design. Considering part warpage as an additional objective results in reductions in material waste of 12 to $17 \%$, these reductions in waste are accompanied by substantial improvements in part quality.
\end{abstract}

Keywords Material waste - Injection molding - Shape optimization $\cdot$ Mesh parameterization $\cdot$ Mold flow analysis · Genetic algorithm

M. Studer $(\bowtie) \cdot$ F. Ehrig

IWK Institute for Materials Sciences and Plastics Processing, University of Applied Sciences, Rapperswil, Switzerland

e-mail: mstuder@hsr.ch

\section{Introduction}

Climate changes and scarcity of resources cause new requirements for product development. Aspects including sustainability and efficiency are becoming increasingly important. Thus, reducing raw material and part weight has gained relevance in the past few years. However, the resulting impact depends strongly on the lot sizes of the parts to be manufactured and on the processes themselves. In particular, in primary shaping processes, such as injection molding, where the material encounters high temperatures, reductions in raw material can also affect the manufacturing time in a positive manner.

Due to the number of boundaries and requirements, the development of new plastic parts for injection molding is challenging. Currently, the concurrent engineering approach (Fig. 1) is the most common in the industry [1]. The strong interconnections between product engineering and product design and the strict use of computer-aided engineering (CAE) accelerate the time-to-market and minimize the development cost [2]. In addition to computer-aided design (CAD), computer-aided molding simulations play the most important role. Such simulations allow for the prediction of the moldability and part quality after molding. In cases of unsatisfactory results, the simulations follow an iteration loop in which the industrial designer optimizes the part design according to amendments proposed by the process engineer.

Such an optimization procedure is time- and costconsuming. With regard to the high complexity, the procedure typically results in numerous iteration loops without a guarantee of finding an optimal solution. Finding the part geometry with the lowest material amount and an acceptable quality is not targeted by using such an approach. To achieve the next generation of concurrent engineering, there 
Fig. 1 Concurrent engineering approach [1]

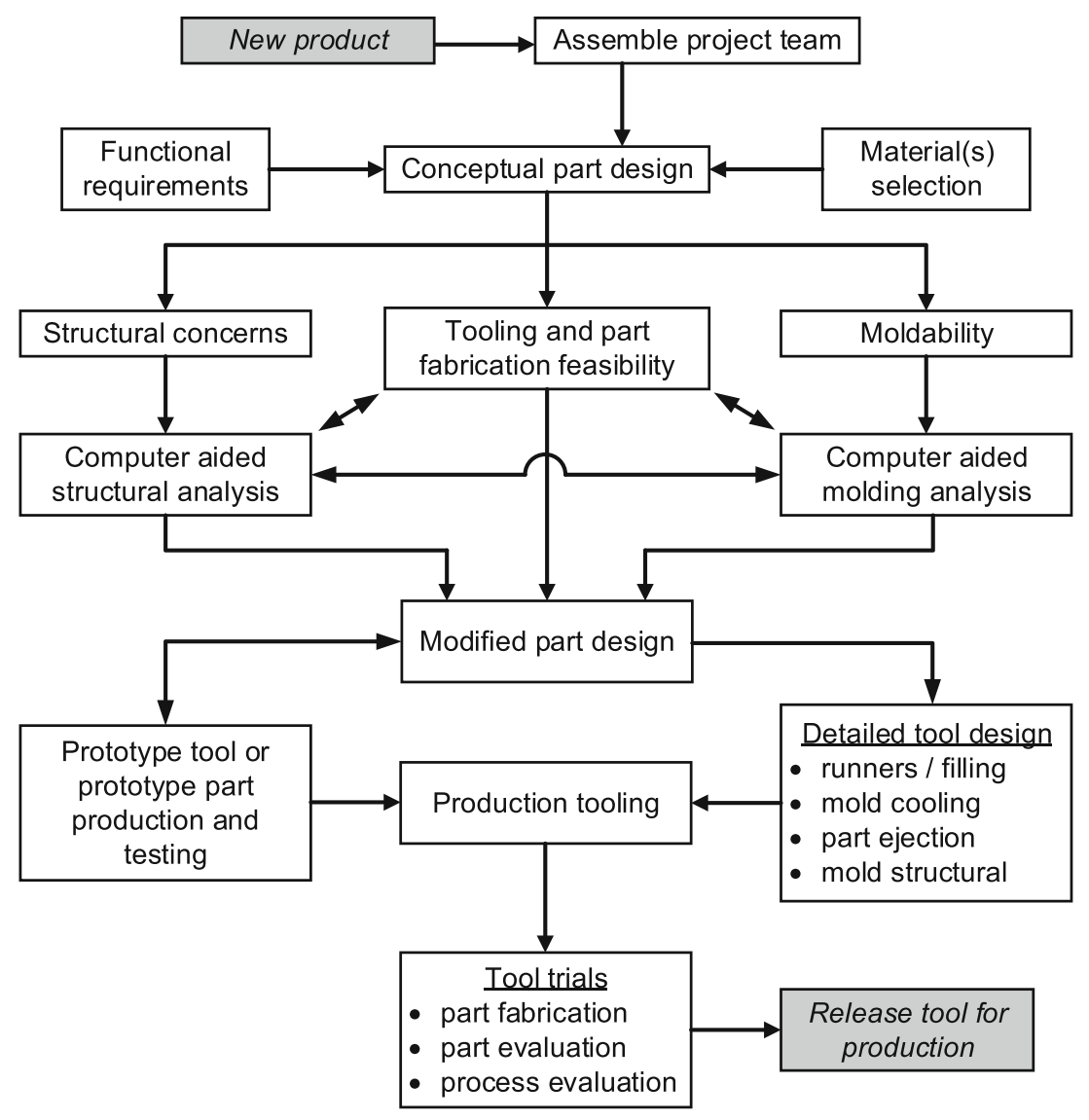

is a need for a new design process in which the part geometry becomes a more active component of computer-aided molding.

Considering the literature, research in actively optimizing geometry based on computer-aided molding software is rare. In 1995, Lee and Kim [3] proposed an optimization routine for minimizing part warpage by adjusting wall thicknesses in different surface selections using discrete values. For this purpose, they used a mid-plane mesh (the wall thicknesses were not geometrically modeled) and optimized the thicknesses using a modified simplex algorithm. Most other studies have focused on the optimization of part quality by adjusting various process variables [4-14] and/or gate locations [15-20].

For this reason, the authors make use of their recently published optimization procedure [21]. The automated procedure consists of a geometry manipulation tool for adjusting the wall thickness distribution based on the surface mesh parameterization technique, a mold flow analysis tool for evaluating the producibility and quality and an evolutionary optimization algorithm for adjusting the wall thickness distribution toward minimum material waste within prescribed tolerances.

In this study, the already developed optimization procedure is for minimizing material waste with respect to producibility and part quality. Therefore, a new objective function is generated, which consists of a measure for the volume of a part. Additional penalty functions ensure the producibility and part quality by limiting the injection pressure to a specific maximum value and considering changes in part warpage during the optimization procedure. The updated procedure is applied on a gearbox platine for a ventilation flap drive and a casing cover for the sanitation industry.

The present paper is organized as follows: Section 2 introduces the proposed optimization procedure and its extension. The realization of the procedure is explained in Section 3. Sections 4 and 5 illustrate its application on a gearbox platine and a casing cover, respectively, to demonstrate its feasibility and robustness. Conclusions and an outlook for future work are provided in Section 6.

\section{Optimization procedure}

The optimization procedure, which was first presented in [21], consists of three main elements: (1) a geometry manipulation tool, (2) a mold flow analysis tool, and (3) an optimization algorithm (Fig. 2). Before reaching a termination criterion, the optimization algorithm creates new design 
Fig. 2 Optimization procedure for optimizing material waste in injection molding [21] with extended data flow

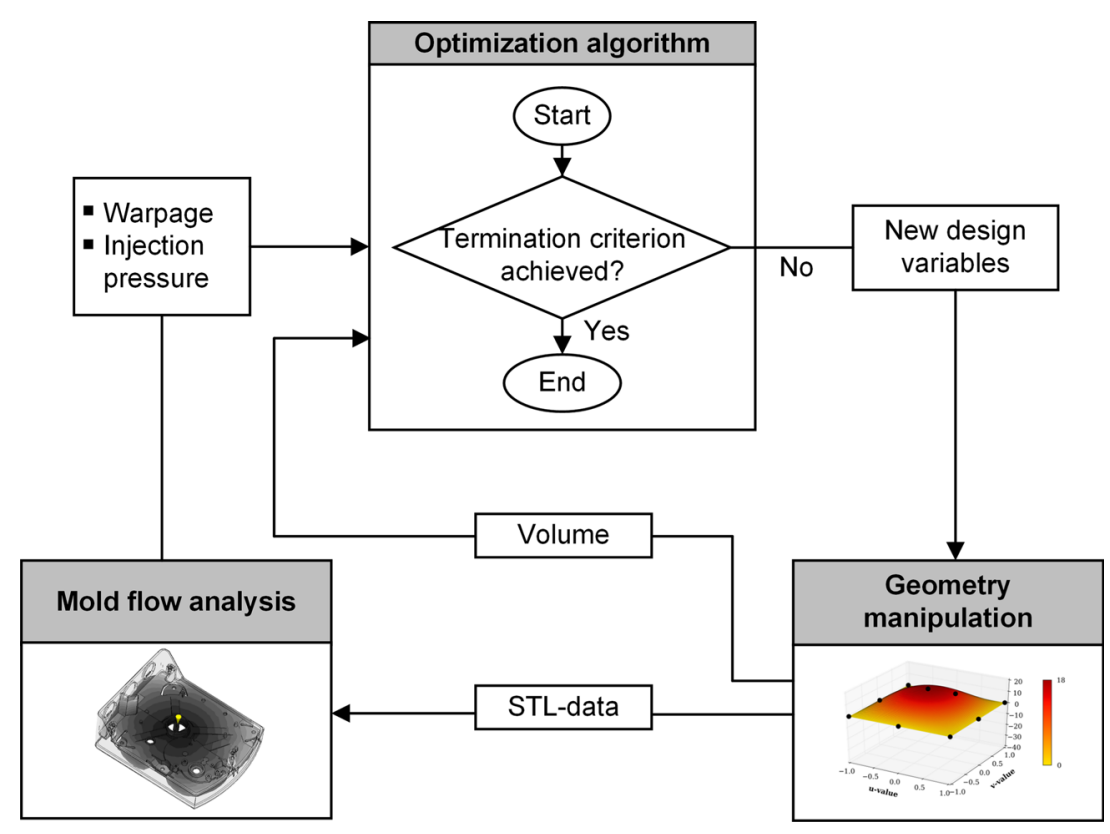

variables and conveys them to the geometry manipulation tool, in which the STL-data of the modified geometry are generated and evaluated using mold flow analysis. Part warpage, injection pressure, and volume values of the actual geometry are transferred to the optimization algorithm. Beginning with the triangulated surface mesh (stereo lithography STL format) of the initial design for the cavity, the procedure results in a surface mesh for the optimized cavity.

\subsection{Geometry manipulation tool}

The geometry manipulation tool is the focal point of the optimization procedure. The tool is based on the unit-square mesh parameterization, which is a widely used technique for computer graphics, surface reconstruction, and remeshing [22]. In computer graphics, this technology is used to apply two-dimensional textures on three-dimensional surfaces. As illustrated in Fig. 3, the surface mesh (A) is parameterized in the unit-square (B), where a texture is applied (C); moreover, based on information from the parameterization, the mesh is transferred onto the surface (D).

Floaters mean value concept [23] has been implemented to receive an nearly shape-preserved parameterization of the surface mesh. Instead of the intended transformation of textures, changes in wall thicknesses are modeled over the unit-square and applied in the direction of the average surface normal at the corresponding nodes on the 3D mesh. The mathematical descriptions for the parameterization and transferring process are provided in [21]. An example of the developed technique on the mannequin is shown in Fig. 4, where the evolutions of two different surface changes for the nose and skull area are demonstrated. Here, the transferring of a thickness distribution with values below zero (i.e., Step 9 in Fig. 4) results in a shrinkage of the skull. Moreover, the modeling of a thickness distribution in the opposite direction for the nose produces an expansion.

Using this technique, changes in wall thickness on arbitrary surface selections are possible with low computational effort. In general, any distribution of thickness changes can be modeled on the unit-square. A generalized polynomial distribution in two dimensions is typically applied (Eq. 1) with an upper limit for the polynomial degree $n_{p}$ of 2 :

$\Delta z(u, v)=\sum_{l=0}^{n_{p}} \sum_{m=0}^{n_{p}} C_{l, m} u^{l} v^{m}$

According to this relationship, constant, bi-linear or biquadratic distributions of thickness changes can be realized.

\subsection{Mold flow analysis}

The commercial product Cadmould 3D-F CMV6 from Simcon kunststofftechnische Software GmbH, Wuerselen, Germany is applied for the mold flow analysis tool. This software solves the equations for the conservation of mass, impulse, and energy based on the simplified equations proposed by Hele-Shaw [24]. Through their patented 3D framework technology for the discretization in the thickness direction [25], melt flow between and across surfaces is calculated, resulting in high computational speed compared to the fully three-dimensional discretization. 
Fig. 3 Illustration of the parameterization technique for applications in computer graphics, which is based on [22]
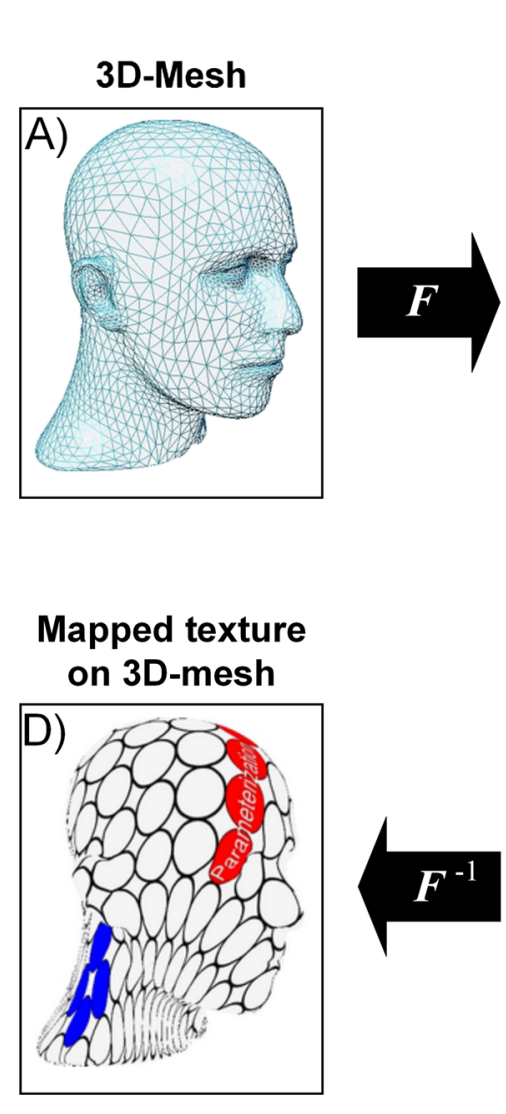

\section{Unit-square parametrization}

B)
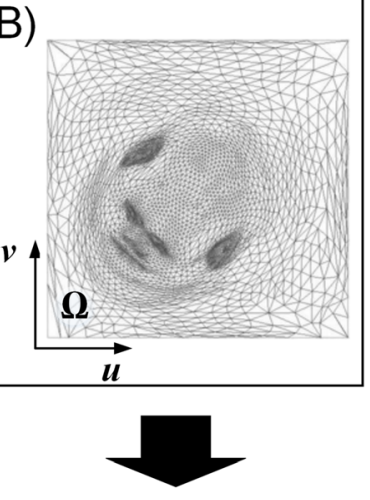

\section{Applied texture}

C)

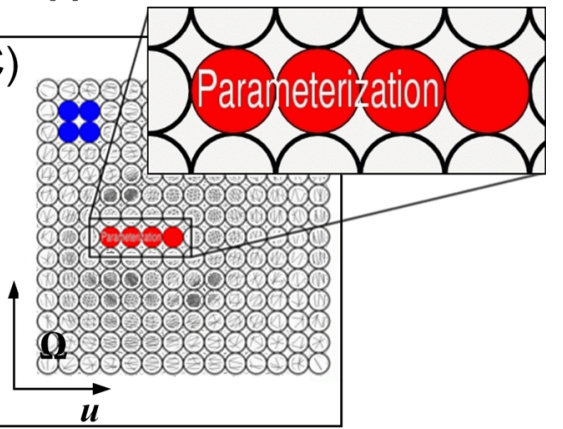

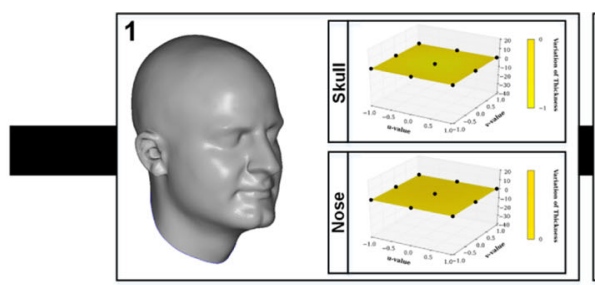
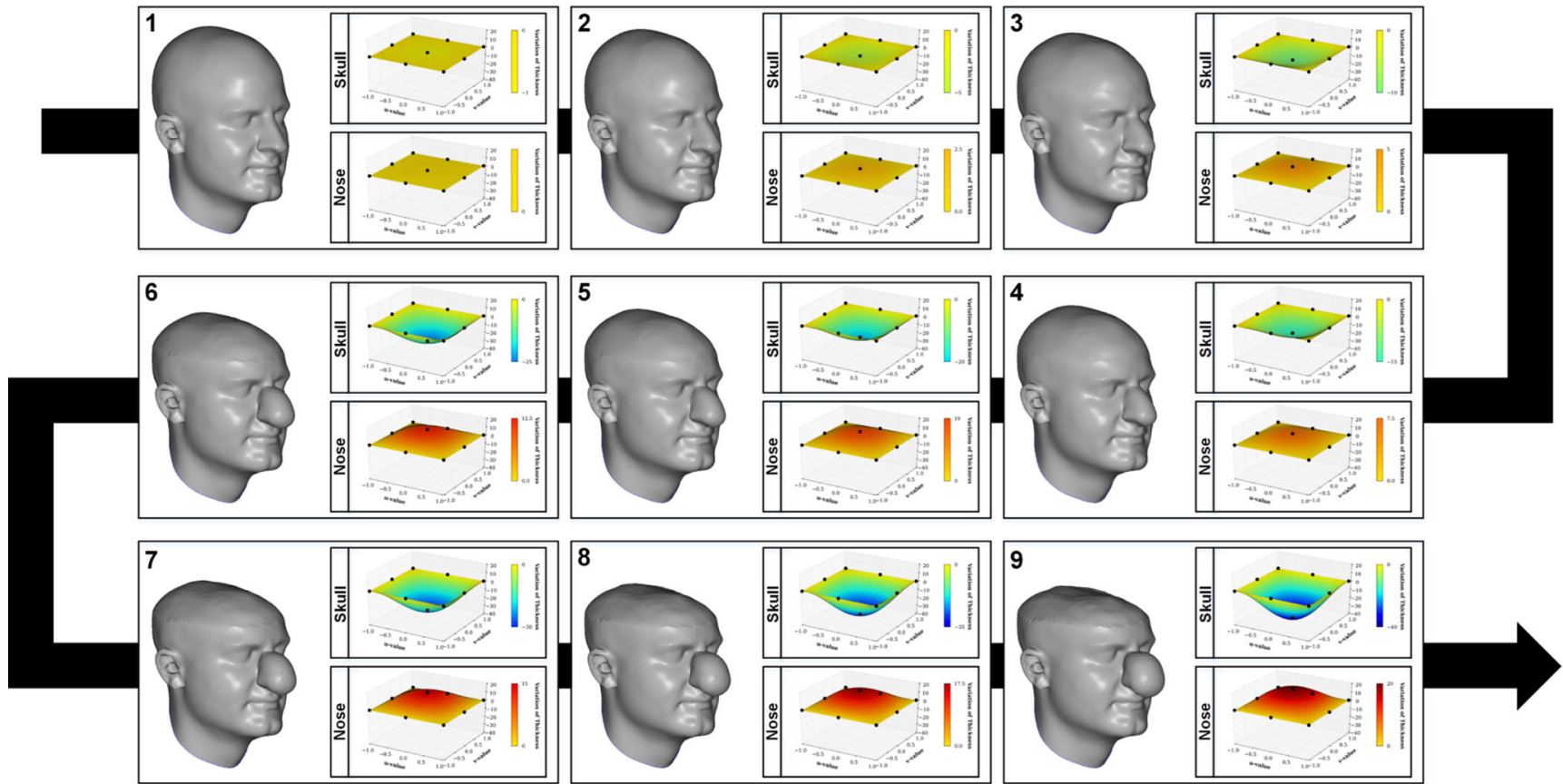

Fig. 4 Illustration of the mesh manipulation using the parameterization technique applied at two different surface selections (nose and skull) 


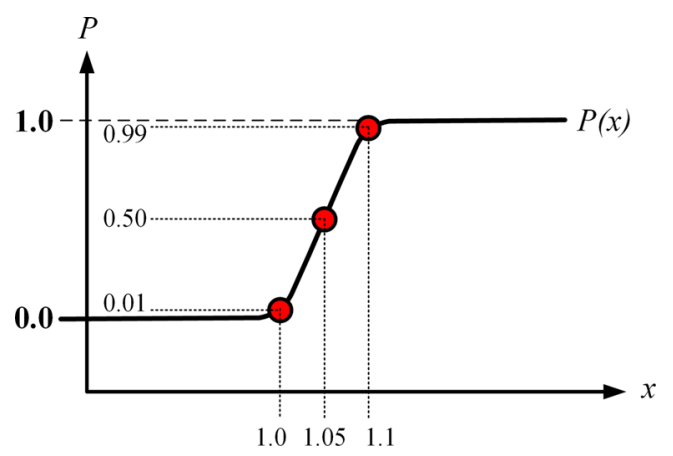

Fig. 5 Sigmoidal penalty function for considering the constraints with an outer bound approach

A viscous-elastic approach is implemented to predict stresses that arise during the cooling of the part inside and outside of the mold. Minimization of the total potential energy leads to associated information regarding deformations [25].

To determine part quality based on warpage, the geometry of the cavity is compared to the geometry of a given molded part. Instead of using the state-of-the-art calculation method, where the geometry of the molded part is compared with the geometry of the isotropic shrunk cavity, a new approach (Eq. 2) has been developed [21]:

$\phi_{w, i}=\angle\left[\mathbf{n}_{\mathbf{c}, \mathbf{i}}, \mathbf{n}_{\mathbf{m}, \mathbf{i}}\right]$

In this approach, an element-wise warpage value is calculated, which considers the angle between the surface normals of the cavity (index c) and the molded part (index m). According to this approach, only out-of-plane deformations are considered.

As mentioned in the introduction, changes in wall thickness can have a significant influence on process conditions. A reduction in thickness requires an increase in the injection pressure $p_{i n}$ and a decrease in the holding pressure time $t_{h}$. To ensure nearly realistic processing conditions during the optimization, the holding pressure and holding pressure time settings are adjusted on every iteration loop according to the actual thickness distribution. For adjusting the material, the specific initial holding pressure $p_{h}^{i n i}$, following the proposed algorithm, is defined:

$$
\text { if } \begin{aligned}
0.8 p_{\text {in }} \leq p_{h}^{\text {ini }} \quad \text { then } \quad p_{h} & =p_{h}^{\text {set }} \\
\text { else } \quad p_{h} & =0.8 p_{\text {in }}
\end{aligned}
$$

This definition ensures that the holding pressure $p_{h}$ reaches at least $80 \%$ of the pressure at the end of the filling $p_{i n}$ and compensates a possible increase in shrinkage resulting from the decrease in pressure transmission.

The modification of the holding pressure time $t_{h}$ is based on Fouriers one-dimensional law of heat conduction. Disregarding the heat produced by the additional melt during the packing stage, the formula reduces to the following expression (Eq. 3):

$t_{h}=t_{h}^{i n i}\left(\frac{s}{s^{i n i}}\right)^{2}$

where the holding pressure time of the actual geometry $t_{h}$ is calculated based on the time for the initial geometry $t_{h}^{i n i}$ multiplied by the squared ratio of the wall thicknesses located close to the gate of the actual $s$ and the initial $s^{i n i}$ geometries.

\subsection{Optimization objectives}

Objectives of optimization For comparison reasons, two different formulations for the objective of optimization are considered. The first formulation $F_{1}$ (Eq. 4) considers the minimization of the volume $V$ as the main objective. The part quality, which is characterized by the average $\phi_{w, a v}$ and maximum $\phi_{w, \max }$ warpage, and the injection pressures at the end of filling $p_{i n}$ are included as additional penalty terms $P(x)$. Therefore, the objective leads to the following expression:

$$
F_{1}=\frac{V}{V^{i n i}}+P\left(\frac{\phi_{w, a v}}{\phi_{w, a v}^{i n i}}\right)+P\left(\frac{\phi_{w, \text { max }}}{\phi_{w, \text { max }}^{\text {ini }}}\right)+P\left(\frac{p_{\text {in }}}{p_{\text {in }}^{\text {max }}}\right)
$$

Fig. 6 Illustration of the binary-coded individual and the genetic operators

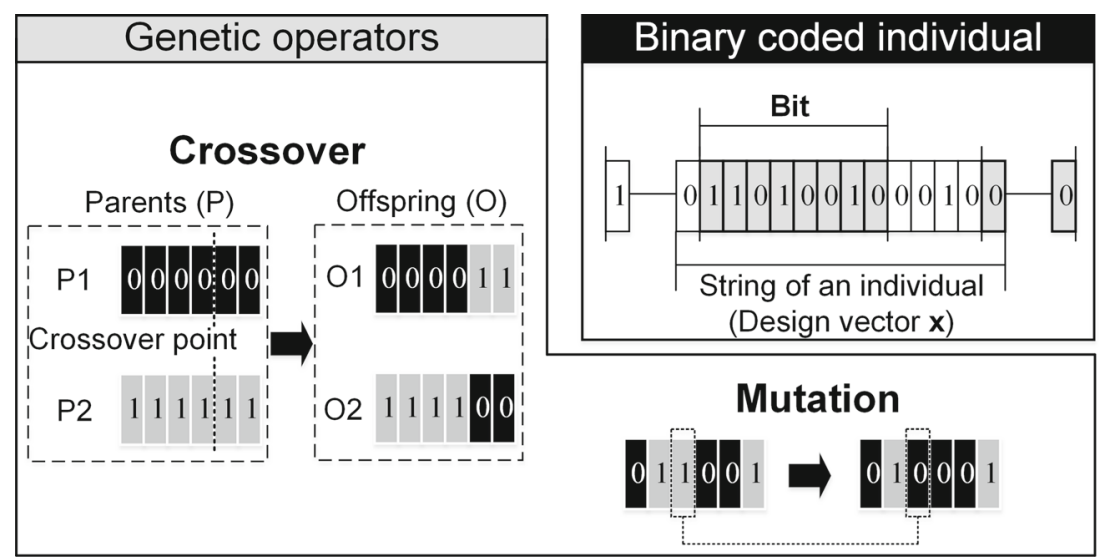


Table 1 Used GA settings

\begin{tabular}{lll}
\hline $\begin{array}{l}\text { Size of } \\
\text { population }\end{array}$ & $\begin{array}{l}\text { Probability } \\
\text { for crossover }\end{array}$ & $\begin{array}{l}\text { Probability } \\
\text { for mutation }\end{array}$ \\
\hline 8 & 0.9 & 0.05 \\
\hline
\end{tabular}

where the average and maximum warpage are calculated as follows (Eq. 5):

$\phi_{w, a v}=\frac{1}{n} \sum_{i=0}^{n} \phi_{w, i} \quad$ and $\quad \phi_{w, \max }=\max \left[\phi_{w, i}\right]$

To achieve the same order of magnitude, the actual volume, warpage, and pressure values at the end of filling are related to the values for the initial design (index ini) or to an upper limit (index $\max$ ). A sigmoidal approach is used for the penalty function (Eq. 6):

$P(x)=\frac{1}{1+e^{-C_{1}\left(x-C_{2}\right)}}$

This widely used approach for structural optimization [26] allows for the modeling of a relatively smooth transition between the limits of zero and one, which is dependent on the coefficient $C_{1}$. The second coefficient $C_{2}$ describes the deflection point, which is where the value of the penalty function reaches 0.5 . Using $C_{1}=91.9$ and $C_{2}=1.05$, the penalty function defines an outer bound, where an increase begins with a function value of $1 \%$ at $x=1.0$ and reaches $99 \%$ of its upper limit at $x=1.1$ (Fig. 5).

To identify, how the minimization of the material waste behaves, if the part quality also is a part of the main objective, a second formulation for the objective function $F_{2}$ is considered (Eq. 7):

$F_{2}=\frac{V}{V^{i n i}}+\frac{\phi_{w, a v}}{\phi_{w, a v}^{i n i}}+\frac{\phi_{w, \max }}{\phi_{w, \text { max }}^{i n i}}+P\left(\frac{p_{\text {in }}}{p_{\text {in }}^{\text {max }}}\right)$

In this formulation (Eq. 7), the constraints for the injection pressure at the end of filling remain the same.
Table 2 Process conditions for the gearbox platine

\begin{tabular}{lll}
\hline Process variable & Unit & Value \\
\hline Injection speed & $\left(\mathrm{cm}^{3} / \mathrm{s}\right)$ & 43.5 \\
Post-fill time & $(\mathrm{s})$ & 30.0 \\
Holding pressure time & $(\mathrm{s})$ & 13.0 \\
Holding pressure & $(\mathrm{MPa})$ & 55.0 \\
Melting temperature & $(\mathrm{deg} C)$ & 300 \\
Surface temperature of cavity & $(\operatorname{deg} \mathrm{C})$ & 95 \\
\hline
\end{tabular}

Optimization problem The objective function can be used to formulate the present optimization problem as follows (Eq. 8):

$$
\begin{array}{rl}
\min _{\mathbf{x} \in \mathbb{R}^{n}} & F(\mathbf{x}) \\
\text { subject to } & \mathbf{l b} \leq \mathbf{x} \leq \mathbf{u b}
\end{array}
$$

where $F$ is the objective function, which is minimized by optimizing the design variables (design vector) $\mathbf{x}$ with respect to upper $\mathbf{u b}$ and lower $\mathbf{l b}$ bounds.

Applied algorithm The lack of an analytical relationship between the design variables $\mathbf{x}$ and the objective function $F$ requires the use of derivative-free optimization (DFO) techniques. Distinguished by the searching algorithm, there exist three primary groups: (1) random search methods, (2) surrogate-based optimization (SBO) methods, and (3) deterministic sampling methods [27]. The results of a previous study [21] in which one representative of each category was applied on low- and highdimensional optimization problems revealed that the deterministic searching method produced the best results for high-dimensional optimization problems (up to a dimension of 18).

Therefore, a genetic algorithm (GA) is used in the current study for solving the optimization problem. This meta-heuristic optimization strategy was developed by
Fig. 7 Gearbox platine with gate location and dark-colored surface selections for the warpage evaluation
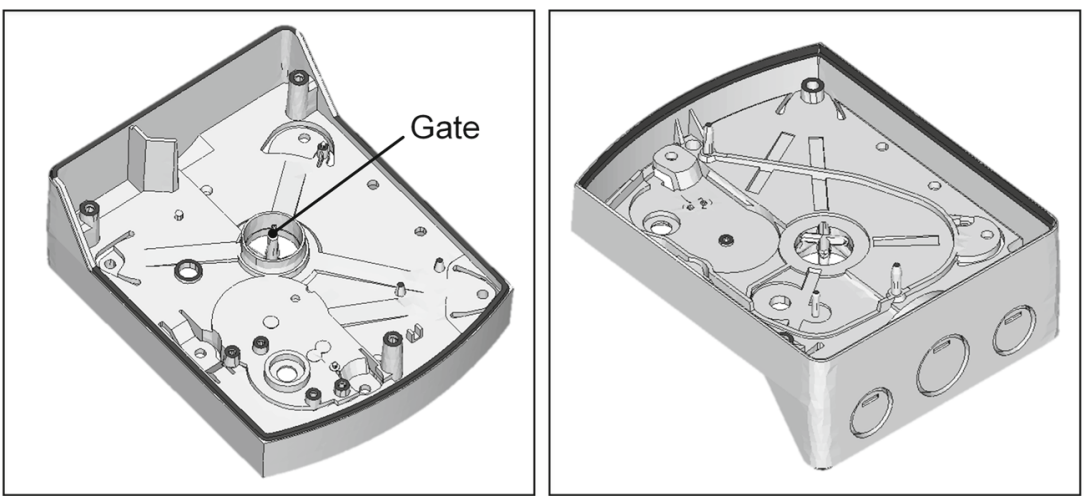
Fig. 8 Surface selections on which the thickness variation is applied
Thickness variation

Bounds Polynomial

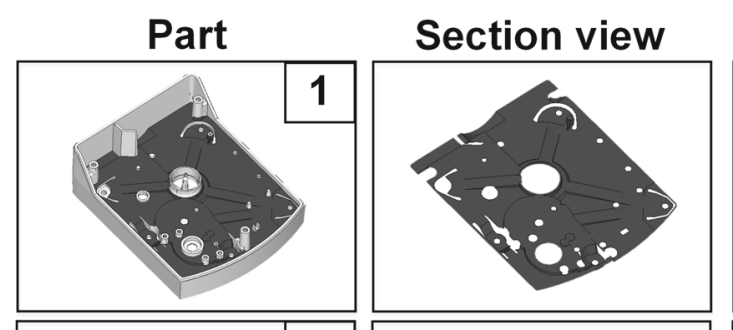

$(\mathrm{mm}) \quad$ degree
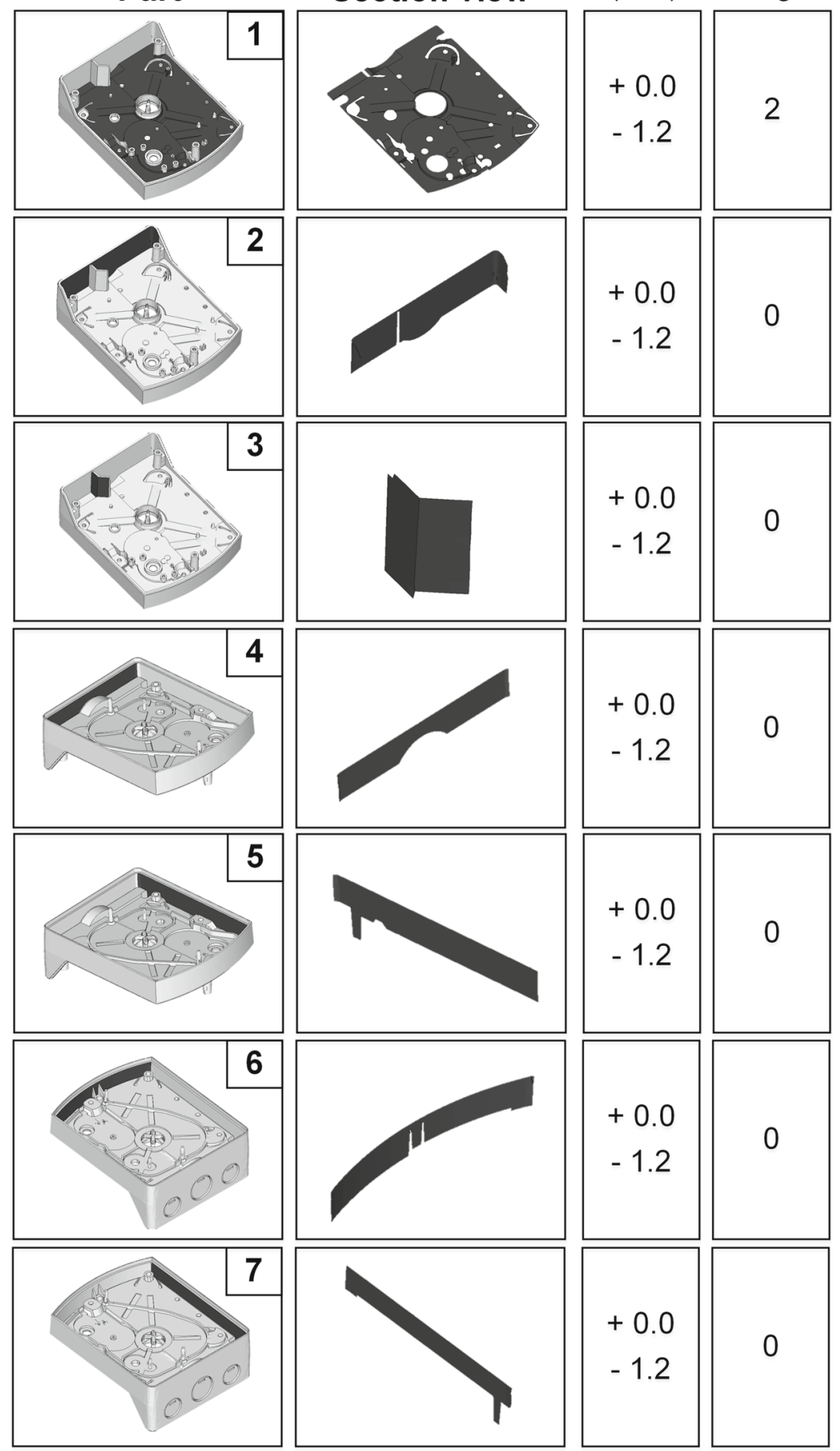

Holland in 1975 [28] and has been successfully applied to many optimization problems in engineering [29]. The procedure simplifies the biological evolution using mathematical imitation of the primary evolution processes, which are summarized as selection and reproduction processes.
For that purpose, a set of individuals (population) and a set of evolutionary operations are generated through which the selected individuals pass on every cycle. Each individual corresponds to the binary string of a design vector $\mathbf{x}$ (Fig. 6). 
Fig. 9 History of optimization for the two different objective functions $F_{1}$ and $F_{2}$ of the gearbox platine with additional illustrations of the ratios of volume $\frac{V}{V^{0}}$, average warpage

$\frac{\phi_{w, a v}}{\phi^{0}, \text { maximum warpage }}$

$\frac{\phi_{w, \max }}{\phi_{w, \max }^{0}}$, and pressure $\frac{p i n}{p_{i n}^{\max }}$
Table 3 Final optimization values for the two different objective functions $F_{1}$ and $F_{2}$ of the gearbox platine

\begin{tabular}{llllll}
\hline$i$ & $F_{i}$ & $\frac{V}{V^{0}}$ & $\frac{\phi_{w, a v}}{\phi_{w, a v}^{0}}$ & $\frac{\phi_{w, \max }}{\phi_{w, \max }^{0}}$ & $\frac{p_{\text {in }}}{p_{\text {in }}^{\text {max }}}$ \\
\hline 1 & 0.71 & 0.71 & 0.74 & 0.81 & 0.93 \\
2 & 1.75 & 0.83 & 0.45 & 0.44 & 1.01 \\
\hline
\end{tabular}

\section{History of optimization for the objective $F_{1}$}
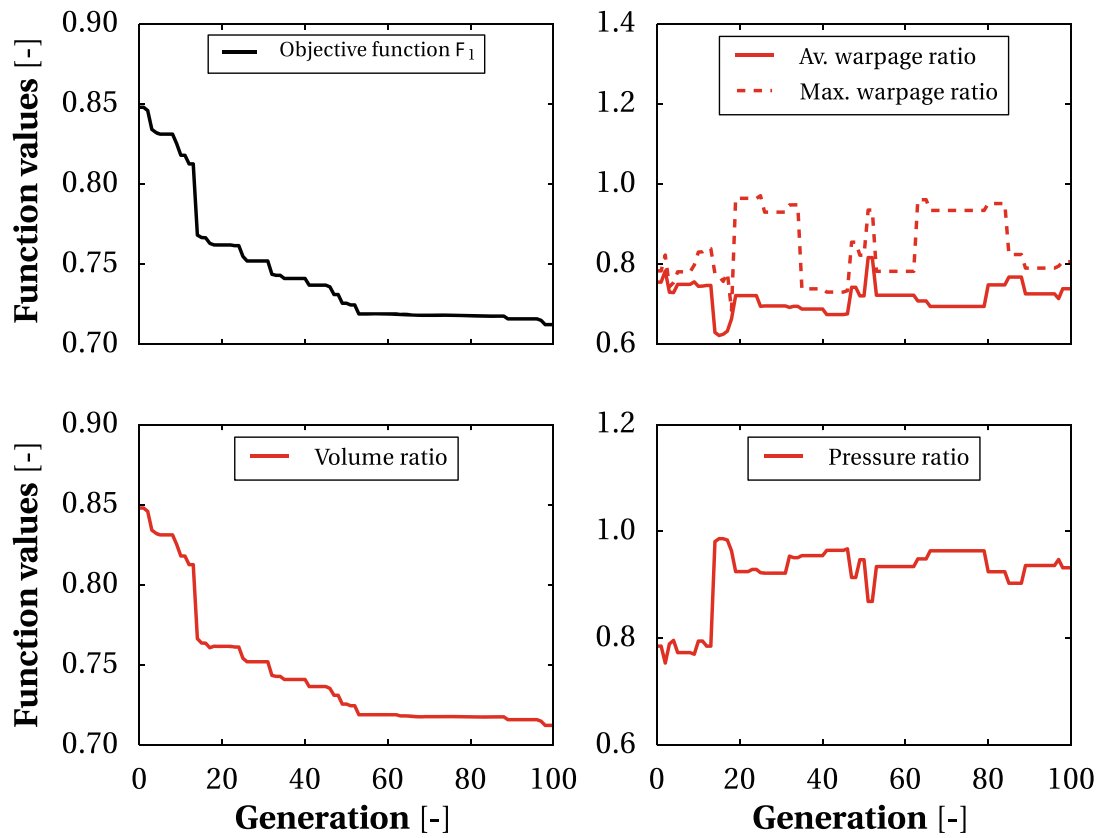

History of optimization for the objective $F_{2}$
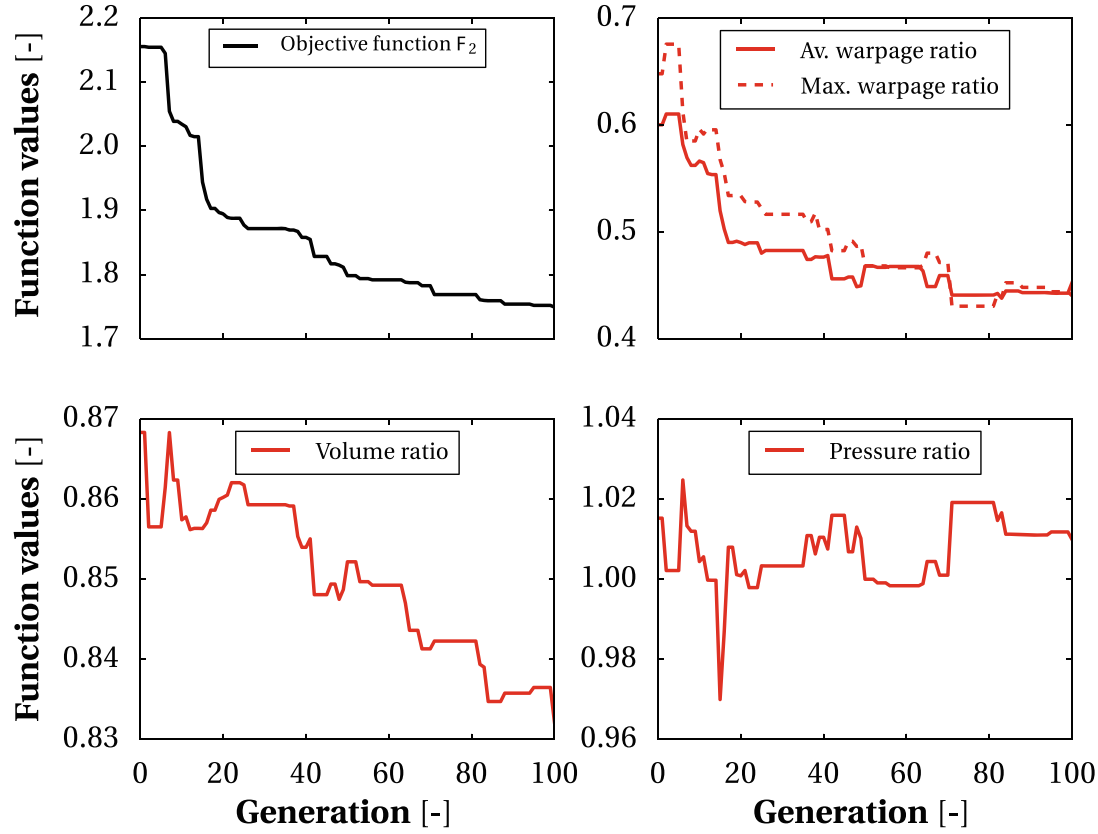

A GA cycle consists of three steps:

1. the evaluation of the present population;

2. the selection of the best individuals; and

3. the production of offsprings by genetic operations.

Crossover and mutation are the primary genetic operators of the reproduction process; at a random position, the binary strings of two selected individuals are recombined (crossover) or inverted (mutation) (Fig. 6). 
Fig. 10 Warpage plot of the initial geometry and the geometries after optimization

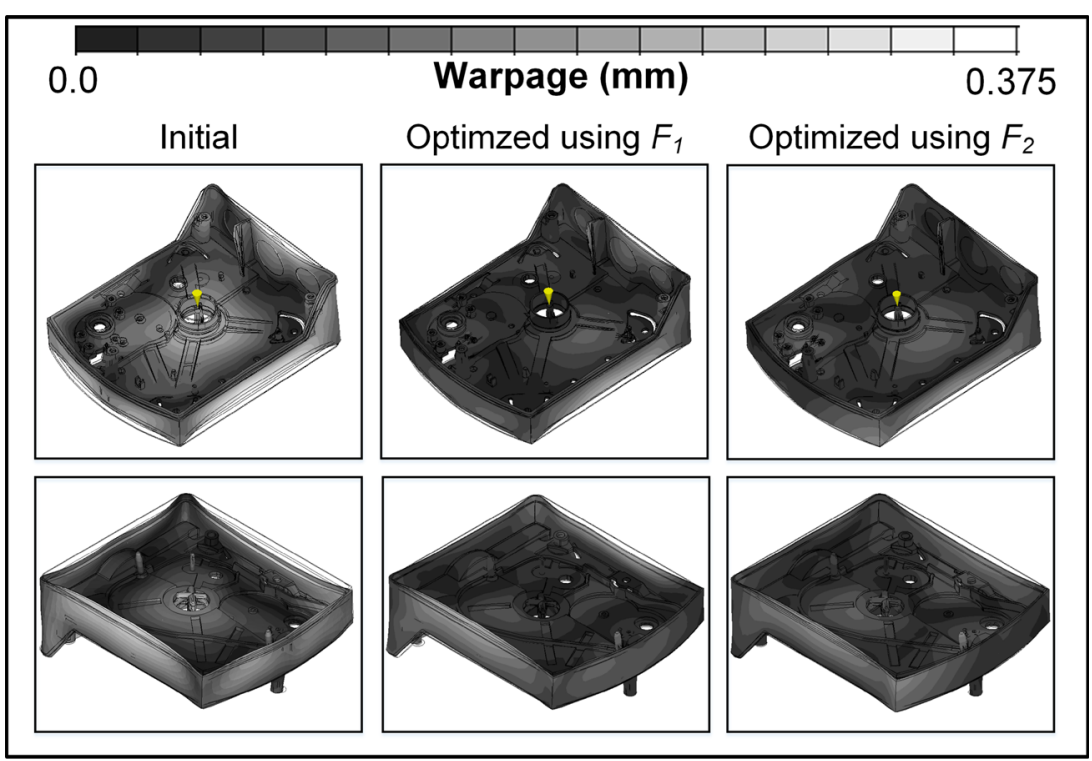

The GA is controlled by only a few parameters:

- the selection method;

- the size of population $s_{p}$;

- the probability for crossover $p_{c}$;

- the probability for mutation $p_{m}$; and

- the termination criterion.

Fig. 11 Comparison of the changes in wall thickness changes according to the two different objective functions (i.e., $F_{1}$ and $F_{2}$ )

\section{Realization of the optimization procedure}

3.1 Implementation and settings

Pyevolve is implemented as a representative for the GA. This evolutionary computation framework is coded in Python. Therein, rank-based selection and the elitism

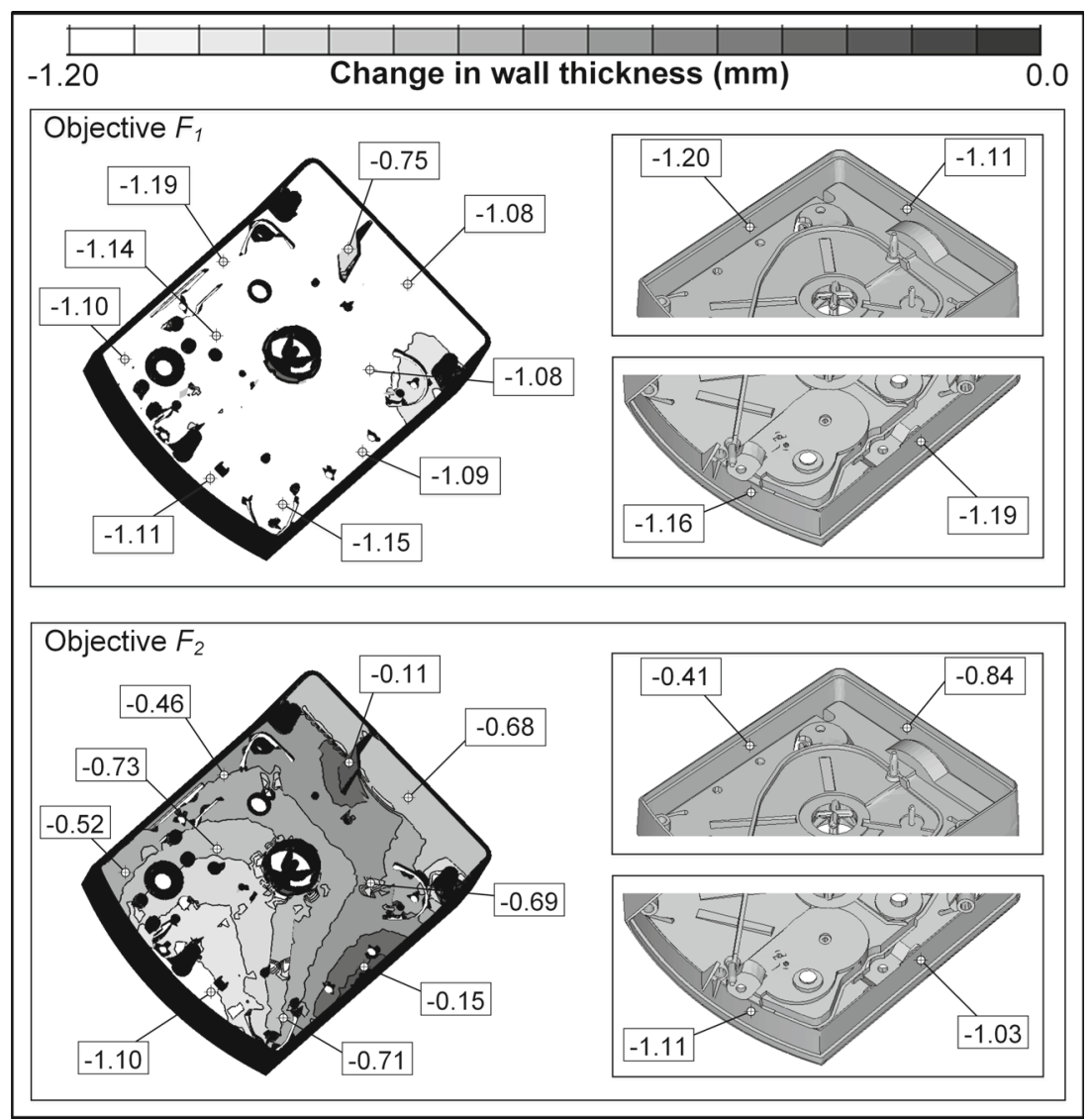


Fig. 12 Casing cover with gate location and dark-colored surface (outer rim) for the warpage evaluation
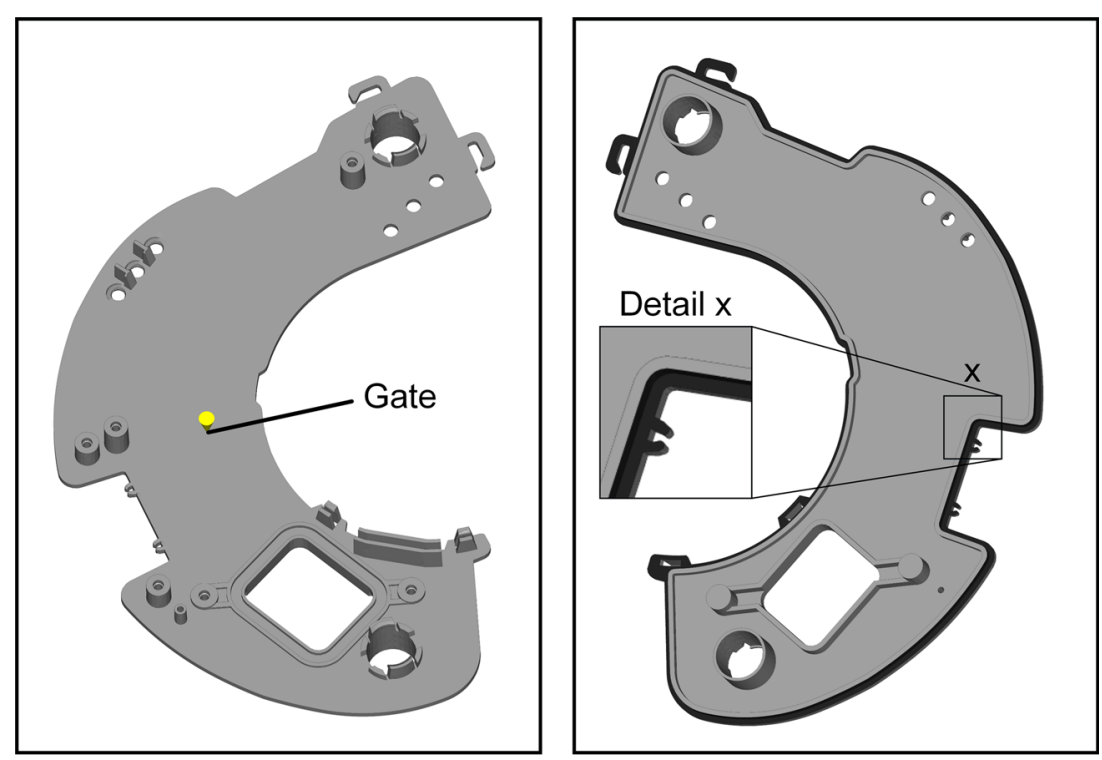

approach, which preserves the fittest individual during the evolution, are utilized. Alleles are defined for maintaining a lower and upper bound on each design variable. An upper limit of 100 cycles (generations) is set as the termination criterion. To demonstrate the usability on state-of-the-art computers, the size of the population (Table 1) is intentionally kept smaller than recommended in the literature [30]. The probabilities for crossover and mutation are listed in Table 1.

The developed geometry manipulation tool is also based on several embedded Python scripts. The optimizations were executed on a $3.5-\mathrm{GHz}$ Intel-i7 processor consisting of 8 cores and $16 \mathrm{~GB}$ of RAM. For the gearbox platine (Section 4), a computation time of approximately $18 \mathrm{~min}$ was required to complete one GA cycle using parallel processing (multi-threading), whereas the casing cover (Section $5)$ required an average time of $4 \mathrm{~min}$. Therefore, the optimizations finished after $30 \mathrm{~h}$ (gearbox platine) and $6 \mathrm{~h}$ (casing cover).

Table 4 Process conditions for the casing cover

\begin{tabular}{lll}
\hline Process variable & Unit & Value \\
\hline Injection speed & $\left(\mathrm{cm}^{3} / \mathrm{s}\right)$ & 49.2 \\
Post-fill time & $(\mathrm{s})$ & 22.0 \\
Holding pressure time & $(\mathrm{s})$ & 15.0 \\
Holding pressure & $(\mathrm{MPa})$ & 60.0 \\
Melting temperature & $(\mathrm{deg} C)$ & 240 \\
Surface temperature of cavity & $(\operatorname{deg} C)$ & 27 \\
\hline
\end{tabular}

\subsection{Workflow of optimization}

The optimization procedure begins with preprocessing. Based on the initial design for the cavity, the following steps are completed:

1. the initial process variables are determined;

2. the areas for thickness optimization are defined; and

3. the parameterization is initiated.

The parameterization is executed only once. The computation times depend strongly on the mesh size. Ordinary mesh sizes require less than a minute of computation time. Afterwards, the primary optimization process is initiated and runs until the termination criterion is reached.

\section{Application on a gear box platine}

\subsection{Input data}

The proposed optimization procedure is first demonstrated on a gearbox platine for a ventilation flap drive (Fig. 7). Its dimensions are $150 \mathrm{~mm} \times 124 \mathrm{~mm} \times 54 \mathrm{~mm}$; the thicknesses of the initial design are between 1.5 and $3.0 \mathrm{~mm}$. Fiber-reinforced polycarbonate (Lexan 1278R) is employed as the polymer material. The process conditions, which are based on the initial design, are listed in Table 2.

As illustrated in Fig. 8, seven different surface selections are defined for the wall thickness optimization procedure. The thickness reductions for all surface selections are limited to a value of $-1.2 \mathrm{~mm}$ due to strength 
Fig. 13 Surface selections on which the thickness variation is applied
Thickness variation

Bounds Polynomial

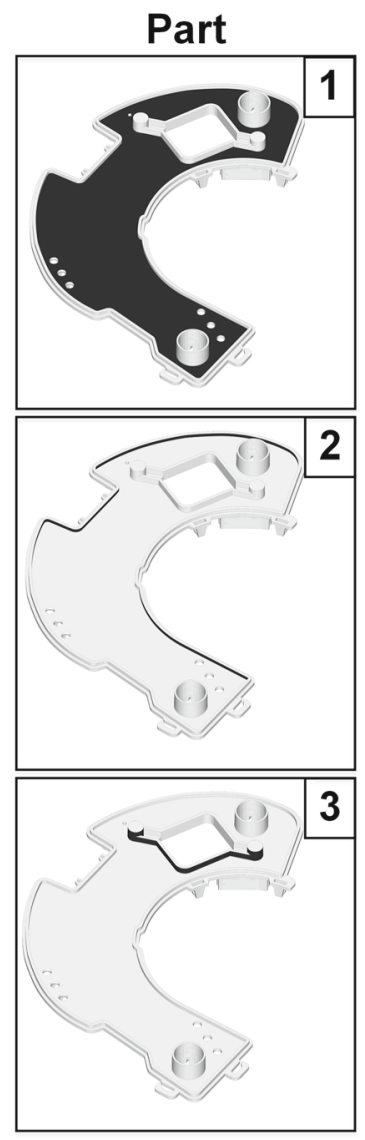

Section view
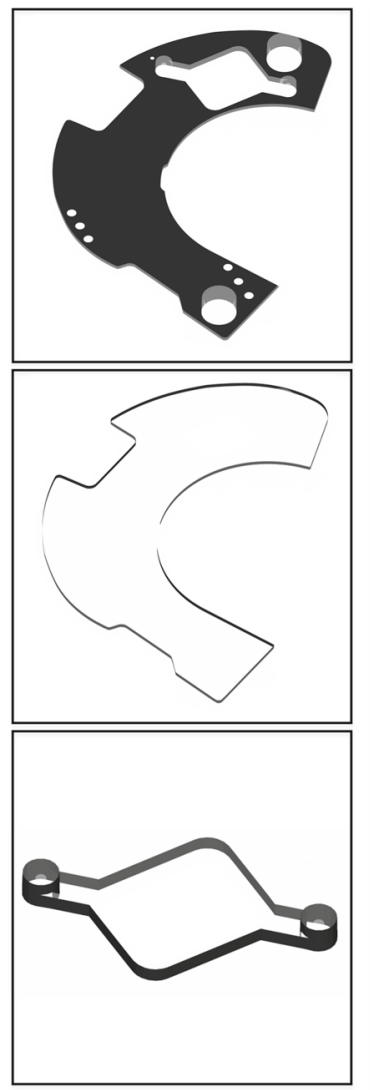

$(\mathrm{mm})$ degree

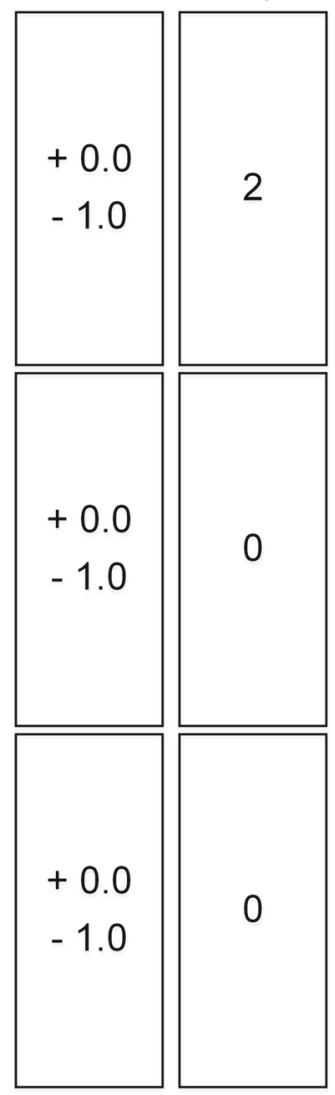

reasons. With the exception of the first surface selection, the polynomial degree is set to 0 , which implies that the thickness changes are constant within those surface selections. Due to the strong influence of melt spreading and pressure transmission on the sidewalls, a polynomial degree of 2 is chose for the bottom area, which enables a biquadratic thickness distribution. Another reason for this choice is due to the high degree of freedom regarding the surface finish and realization.

By applying those settings for the wall thickness variations, the design vector $\mathbf{x}$ consists of $9+6$ design variables, i.e., 9 for manipulating the thickness distribution at surface selection 1 and 1 each for adjusting the thickness in the other 6 surface selections.

The surface selections used for evaluating part quality, which are characterized by warpage, correspond to the dark-colored areas in Fig. 7. Based on the assembly after molding, the interfaces and positioning elements, such as the upper and lower rim of the gear housing and the upper edge surfaces of the screw bosses, are considered.
To prevent excessive increases in the clamping force and to ensure the producibility with standard injection units, the maximum permissible pressure at the end of filling $p_{i n}^{\max }$ is set to $100 \mathrm{MPa}$.

\subsection{Results and discussion}

Comparison of the objectives The history of optimization for both objective functions $F_{1}$ and $F_{2}$ is illustrated in Fig. 9 with the representation of each portion of the objectives. Considering the value for the objective function, both formulations rapidly decrease during the first fifth of the completed generations. By comparing the two objectives, $F_{1}$ and $F_{2}$ presented in Fig. 9, the part quality evolution, which is characterized by the ratio of the averaged and maximum change in surface normals between the actual and the initial geometry, and the volume evolution are different.

While part warpage in the first objective $F_{1}$ is not an active optimization component, the resulting part warpage history exhibits a strong fluctuation; the values vary 
Fig. 14 History of optimization for the two different objective functions (i.e. $F_{1}$ and $F_{2}$ ) of the casing cover with additional illustrations of the ratios of volume $\frac{V}{V^{0}}$, average warpage $\frac{\phi_{w, a v}}{\phi_{w, a v}^{0}}$, maximum warpage $\frac{\phi_{w, \max }}{\phi_{w, \max }^{0}}$ and pressure $\frac{p i n}{p_{i n}^{\max }}$

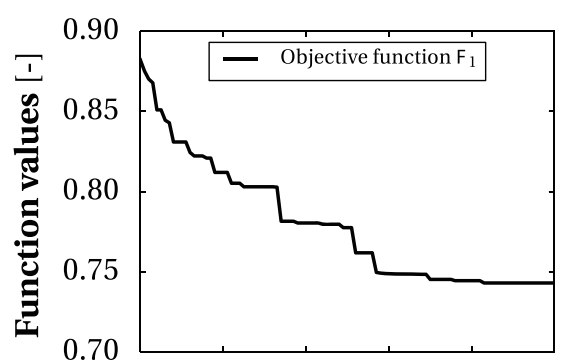

History of optimization for the objective $F_{1}$
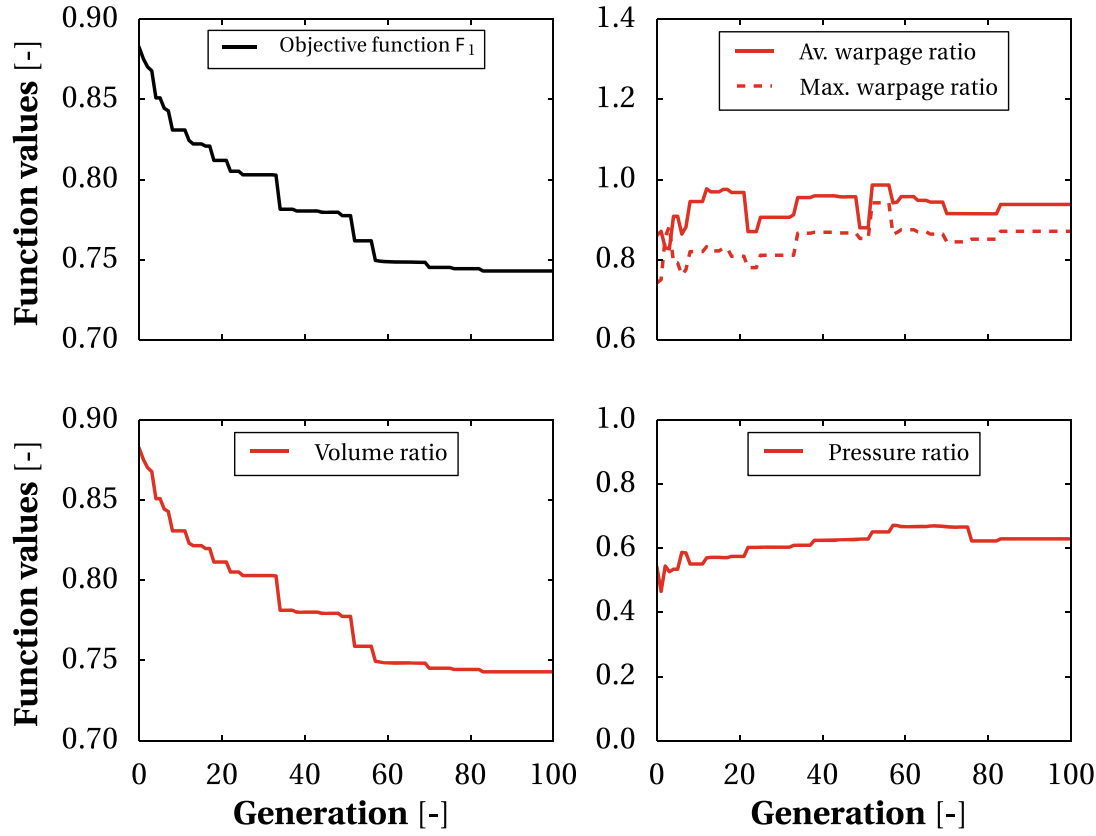

History of optimization for the objective $F_{2}$
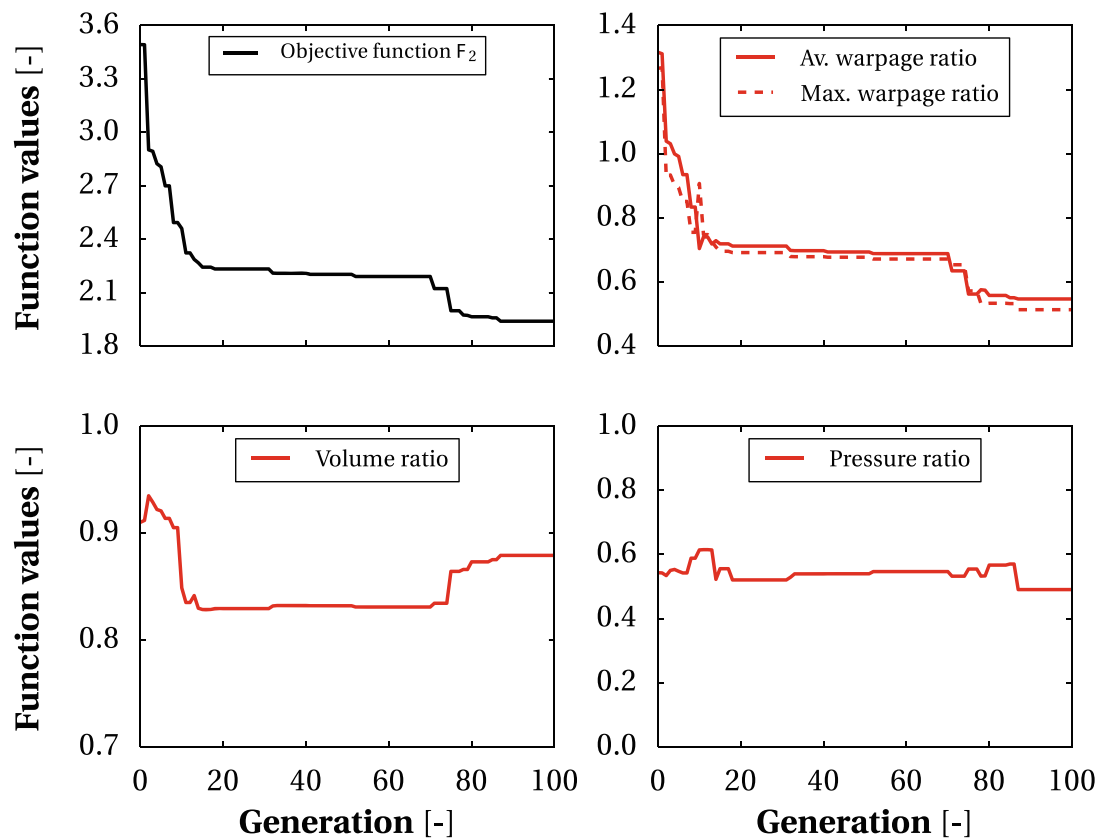

between an upper value, which occurs shortly before the penalty function becomes active, and a lower value of 0.6. The use of part quality as an additional main objective in objective function $F_{2}$ results in a significant and continuous decrease in warpage.

Comparing the evolution of the volume ratio, the influence of including part quality as an active component in objective function $F_{2}$ is observed. While the isolated activation of the volume $\left(F_{1}\right)$ results in a volume reduction of nearly $30 \%$, the additional considered part quality achieves reductions of only approximately $17 \%$ (Table 3 ).

Considering the pressure ratio, both optimizations force the pressure to increase to the upper limit immediately before the penalty function becomes active. Thus, both formulations guarantee the adherence of the specified pressure limit. 
Table 5 Final optimization values for the two different objective functions (i.e., $F_{1}$ and $F_{2}$ ) of the casing cover

\begin{tabular}{llllll}
\hline$i$ & $F_{i}$ & $\frac{V}{V^{0}}$ & $\frac{\phi_{w, a v}}{\phi_{w, a v}^{0}}$ & $\frac{\phi_{w, \max }}{\phi_{w, \max }^{0}}$ & $\frac{p_{i n}}{p_{i n}^{\text {max }}}$ \\
\hline 1 & 0.74 & 0.74 & 0.94 & 0.87 & 0.63 \\
2 & 1.94 & 0.88 & 0.55 & 0.51 & 0.49 \\
\hline
\end{tabular}

Visualization of the results To better classify the part quality results and thickness changes, plots of deformations and changes in the wall thickness are compared. The warpage plot in Fig. 10, in which the deviations between the molded part and the isotropically shrunk geometry for the cavity are visible, illustrates the part quality results. All of the geometries are plotted using an identical color range and identical scaling factor of 20 for the resulting deformations. Compared to the initial cavity geometry, both optimizations lead to an improvement. The considerable improvement of objective function $F_{2}$ compared to objective function $F_{1}$ corresponds well with the values in Table 3.

Changes in wall thickness are presented in Fig. 11. Changes between the initial and the optimized geometries according to the two different objective functions (i.e., $F_{1}$ and $F_{2}$ ) are colored and denoted at specified points. Figure 11 shows that using the first objective function, in which only the volume is considered as an active optimization variable, results in thicknesses that tend toward the lower limit of $-1.2 \mathrm{~mm}$ in nearly all surface selections. Using the second objective $\left(F_{2}\right)$ with the additional consideration of part quality results in a broader distribution in the resulting wall thickness changes. This result reveals that optimizing part quality is due to a specified distribution of the wall thickness, which corresponds to the findings in a previously published study [21].

\section{Application on a casing cover}

\subsection{Input data}

For the second example, a casing cover used in the sanitation industry is considered (Fig. 12). Its dimensions are 250 $\mathrm{mm} \times 203 \mathrm{~mm} \times 28 \mathrm{~mm}$; the main wall thickness of the initial design is $2.5 \mathrm{~mm}$. Unreinforced ABS Terluran GP-22 is employed as the polymer material. The process conditions, which are obtained based on the initial design, are listed in Table 4.

For optimizing wall thicknesses toward minimal material waste, three different surface selections are considered (Fig. 13). Selection 1 forms the main part of the cover's surface; therefore, this component primarily influences filling and consolidation. For achieving a high variability in this selection, a polynomial degree of 2 is applied for the thickness variation. Surface selection 2 influences the thickness of the rip along the opening, while selection 3 includes the thickness of the surrounding rip. For both, a polynomial degree of zero is used, which allows only uniform changes in the thickness.

These settings result in a design vector that consists of $9+1+1$ variables for the optimization. According to the structural requirements, all thickness changes are limited to a value of $-1.0 \mathrm{~mm}$. During the subsequent assembly process, the cover undergoes a welding process in which a second part is attached to the outer rim. Therefore, warpage of the outer rim should be as low as possible and is selected as the quality criterion for optimization (dark-colored areas in Fig. 12).

In reality, the casing cover is molded using a hot runner system. Figure 12 illustrates the specified location of the gating point. As for the gearbox platine, the casing cover is discretized using an average finite element mesh size of $2 \%$ of the space diagonal to the geometry.
Fig. 15 Warpage plot for the initial geometry and the geometries after optimizing

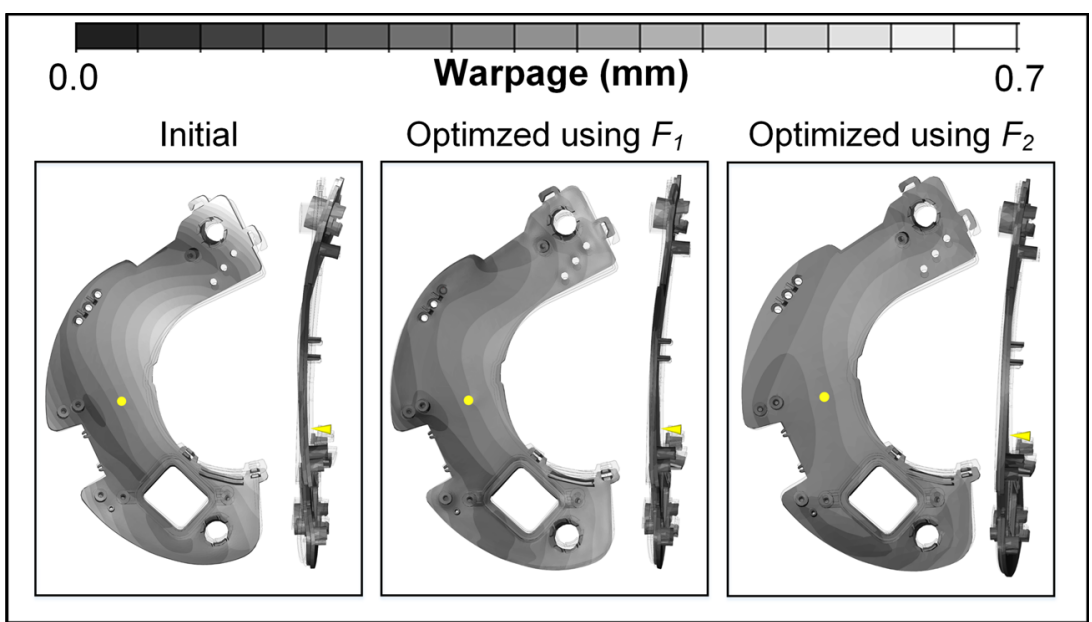


Fig. 16 Comparison of the wall thickness changes at surface selection 1 according to the two different objective functions (i.e., $F_{1}$ and $F_{2}$ )

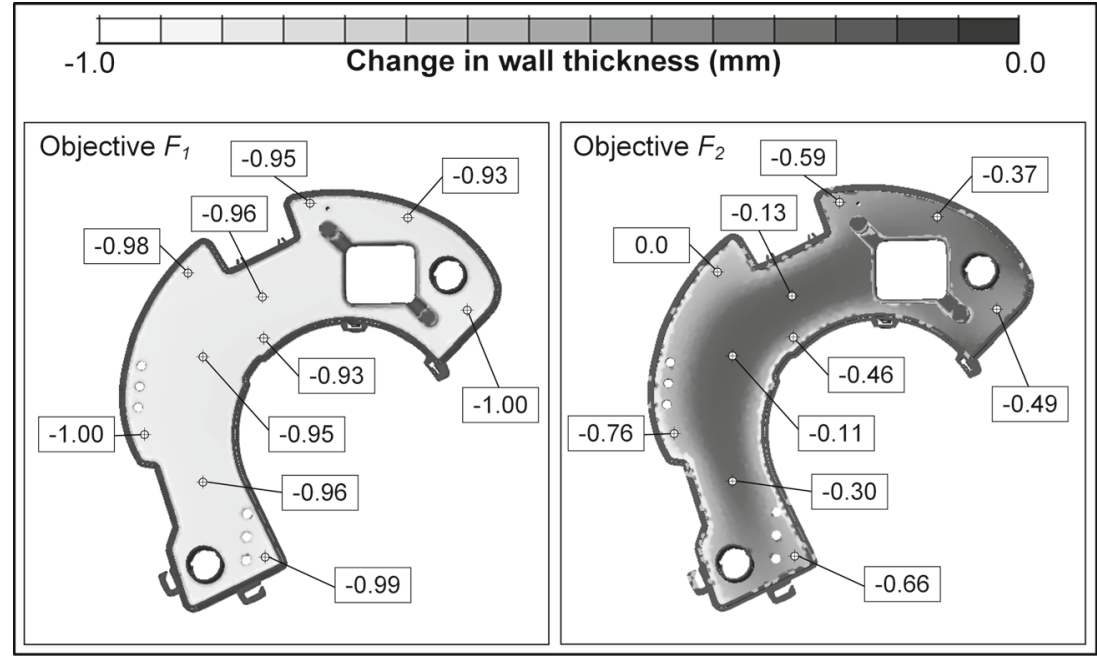

The corresponding finite element mesh consists of approximately 10,145 triangular elements.

\subsection{Results and discussion}

Comparison of the objectives As for the gearbox platine, the optimization histories for both objective functions (i.e., $F_{1}$ and $F_{2}$ ) are compared. Figure 14 illustrates the results for each objective and their components separately. Considering the two different objective functions, both show a large decrease and tend to reach a minimum value after completing 100 GA-cycles. The warpage evolution shown in Fig. 14 demonstrates the different behaviors during the optimization. Whereas the passive consideration of warpage in $F_{1}$ again tends to the upper bound, $F_{2}$ minimizes both warpage values.

By considering the volume ratio history, objective function $F_{1}$ results again in a significant decrease, whereas objective function $F_{2}$ begins with a decrease in the volume ratio during the first generations followed by an increase after 78 generations.

The pressure ratio history reveals that both optimization strategies do not reach the upper pressure limit of 100 MPa. Table 5 shows the optimized values for the different components of the objective functions. Accordingly, the objective function that passively considers part quality $\left(F_{1}\right)$ results in a volume reduction of approximately $26 \%$. By considering part warpage as an active component $\left(F_{2}\right)$, the volume reduction is only $12 \%$, which is accompanied by a decrease in part warpage of 45 and $49 \%$.

Visualization of the results The part warpage results, which are characterized by the geometrical deviation between the molded part and the isotropically shrunk cavity, are presented in Fig. 15 using the same color scale and a geometrical scaling factor of 20. Here, the color ranges and deformed geometries confirm the values presented in Table 5, demonstrating the optimization using $F_{2}$ results in the smallest part warpage. Finally, the changes in wall thickness at surface selection 1 between the initial and the optimized geometries are presented in Fig. 16.

The light gray surface of the optimized geometry using objective function $F 1$ illustrates that the thickness tends to the lower limit of $-1.0 \mathrm{~mm}$ over the entire region. For objective function $F_{2}$, the optimization again results in a non-uniform wall thickness distribution in which the maximum values are located near the gate. From such a distribution follows an improved pressure transmission and a reduced potential of shrinkage at locations far from the gate. This distribution reduces the potential of inhomogeneous shrinkage [21] and the potential for warpage.

\section{Conclusions}

In this study, a new method for optimizing the amount of material required for injection-molded parts is presented. The method is based on a numerical optimization routine in which an optimization algorithm is connected to a geometry manipulation tool and a mold flow analysis. The active manipulation of the geometry by adjusting its wall thicknesses at user-defined surface selections within prescribed tolerances and the ongoing evaluation of its moldability and part quality allows the development of new products in an advanced manner, which will minimize time- and cost-consuming iteration loops.

The application of the procedure on two different industrial parts demonstrates the practical applicability because of low user input, computational times of less than $30 \mathrm{~h}$ on a state-of-the-art personal computer, reductions in material 
waste of up to $30 \%$ when simultaneously maintaining part quality.

As demonstrated on both geometries, the amount of reduced material depends on the quality requirements of the molded parts. Simultaneously, minimizing material waste and part quality results in reduced material savings than considering only part quality as a passive boundary condition. Thus, optimizing part quality and material savings can result in conflicting interests.

However, the advantages of the presented approach are the fast, process-specific, and CAD-software-independent parameterization of the geometry; however, these benefits come with the disadvantage of the lack of a seamless update in the origin solid model. The used parameterization technique is predestined for applications on areal parts. Geometries with numerous complex surface sections may lead to numerous design variables, which cannot be solved efficiently on a state-of-the-art computer.

The presented methodology will be developed further. Major research topics will include the prevention of possible undercuts and user-defined weighting of the objectives for material waste and part quality.

Acknowledgements The authors wish to thank Simcon Corporation and the open-source projects Python, Scipy, Pyevolve, Matplotlib and GOM-Inspect, for making their software available for this study.

\section{References}

1. Malloy RA (2010) Plastic part design for injection molding: an introduction. Carl Hanser, Munich

2. Albers A, Nowicki L (2003) Integration der simulation in die produktentwicklung, symposium, Bremen

3. Lee BH, Kim BH (1995) Optimization of part wall thicknesses to reduce warpage of injection-molded parts based on the modified complex method. Polym Plast Technol Eng 34:793-811

4. Yin F, Mao H, Hua L, Guo W, Shu M (2011) Back propagation neural network modeling for warpage prediction and optimization of plastic products during injection moulding. Mater Des 32:1844-1850

5. Shi HZ, Gao YH, Wang XC (2010) Optimization of injection molding process parameters using integrated artificial neural network model and expected improvement function method. Int $\mathbf{J}$ Adv Manuf Technol 48:955-962

6. Deng YM, Zhang Y, Lam YC (2010) A hybrid of mode-pursuing sampling method and genetic algorithm for minimization of injection molding warpage. Mater Des 31:2118-2123

7. Farshi B, Gheshmi S, Miandoabchi E (2011) Optimization of injection molding process parameters using sequential simplex algorithm. Mater Des 32:414-423

8. Kurtaran H, Ozcelik B, Erzurumlu T (2005) Warpage optimization of a bus ceiling lamp base using neural network model and genetic algorithm. J Mater Process Technol 169:314-319
9. Ozcelik B, Sonat I (2009) Warpage and structural analysis of thin shell plastic in the plastic injection molding. Mater Des 30: 367-375

10. Huang MC, Tai CC (2001) The effective factors in the warpage problem of an injection molded part with a thin shell feature. $\mathbf{J}$ Mater Process Technol 110:1-9

11. Kong WY, Kim JK (2003) Warpage in plastic packages: Effects of process conditions geometry and materials. IEEE Trans Compon Packag Manufact 26:2345-2352

12. Erzurumlu T, Ozcelik B (2006) Minimization of warpage and sink index in injection-molded thermoplastic parts using Taguchi optimization method. Mater Des 27:853-861

13. Wang GL, Zhao GQ, Li HP, Guan YJ (2009) Influence factor analysis of warpage and optimization of process parameters in plastic injection molding of thin-wall plastic parts based on Taguchi optimization method. China Mech Eng 20:488-492

14. Gao YH, Wang XC (2008) An effective warpage optimization method in injection molding based on the Kriging model. Int $\mathbf{J}$ Adv Manuf Technol 37:953-960

15. Zhai M, Xie Y (2010) A study of gate location optimization of plastic injection molding using sequential linear programming. Int J Adv Manuf Technol 49:97-103

16. Pandelidis I, Zhou Q (2004) Optimization of injection molding design. Part I: gate location optimization. Polym Eng Sci 30:873882

17. Zhai M, Shen CY (2005) An optimization scheme based on flow resistance to locate optimum gate of complex part. J Reinf Plast Compos 24:1559-1566

18. Zhai M, Lam YC, Au CK, Liu DS (2005) Automated selection of gate location for plastic injection molding processing. Polym Plast Technol Eng 44:229-242

19. Lam YC, Jin S (2001) Optimization of gate location for plastic injection moulding. J Inj Molding Technol 5:180-192

20. Lam YC, Britton GA, Liu DS (2004) Optimization of gate location with design constraints. Int J Adv Manuf Technol 24: 560-566

21. Studer M, Ehrig F (2014) Minimizing part warpage in injection molding by optimizing wall thickness distribution. J Adv Pol Technol. Early View doi:10.1002/adv.21454

22. Yoshizawa S, Belayev A, Seidel HP (2004) A fast and simple stretch-minimizing mesh parameterization. Proc Shape Model Appl 8:200-208

23. Floater MS (2003) Mean value coordinates. Comput Aided Geom Des 20(1):19-27

24. Hele-Shaw HS (1899) The motion of perfect liquid. Proc Royal Inst 16:49-64

25. Filz PF, Kutschera H, Stoeckmann H, Welter F, Webelhaus K (2006) Simulation of fluid flow and structural analysis within thin walled three dimensional geometries. Eur Pat 1,385,103

26. Kress G, Keller D (2007) Structural optimization, lecture Script. Swiss Federal Institute of Technology Zurich, Switzerland

27. Hemker $\mathrm{T}$ (2008) Derivative free surrogate optimization for mixed-integer nonlinear black box problems in engineering, Ph.D. thesis, Technical University Darmstadt, Germany

28. Holland J (1975) Adaption in natural and artifical systems. The University of Michigan press, Ann Arbor, USA

29. Goldberg D (1989) Genetic algorithms in search optimization and machine learning. Addison-Wesley, Massachusetts, USA

30. Nissen V (1997) Einfuehrung in evolutionaere algorithmen. Vieweg und Teubner Verlag, Germany 